\title{
PRESENTAZIONE DEL SYMPOSIUM
}

\author{
GIANNANTONIO SACCHI LANDRIANI (*)
}

Ho davanti il primo numero di Meccanica del settembre 1966 e l'ultimo (il n ${ }^{\circ}$ ) dell'ottobre 2016, e i numeri del 1966 e l'ultimo uscito (2014) dei Rendiconti, pubblicazione annuale, dell'Istituto Lombardo Accademia di Scienze e Lettere.

Meccanica, organo ufficiale di AIMETA, compie 50 anni. È una rivista di grande successo, nata con quattro numeri per anno, con l'intento di far conoscere in sede internazionale il lavoro scientifico di ricercatori italiani.

Tra AIMETA ed Istituto Lombardo c'è stato fin dall'origine della rivista un forte legame testimoniato dalla presenza dei promotori dell'associazione di buona parte di membri del Lombardo. Infatti nel primo "Comitato di Redazione" del 1966 figurano: Capocaccia, Ferrari, Bruno e Leo Finzi, Graffi, Luigi Napolitano, Supino. Direttore Responsabile Emilio Massa, Segretario di Redazione Giovanni Bianchi.

I temi proposti sono relativi alla meccanica in senso lato: Meccanica generale, Termodinamica, Meccanica dei solidi e delle strutture, Meccanica razionale e superiore, Fisica matematica, Meccanica applicata alle macchine, Scienza e Tecnica delle costruzioni, Meccanica dei fluidi, Idraulica, Aerodinamica, Gasdinamica, Meccanica celeste.

Nel primo numero figurano 11 contributi tutti di autori italiani tra i quali figurano Bruno Finzi, Grioli, Ferrari, Zanaboni.

Sfogliando Meccanica dalle origini ad oggi si rileva che ogni numero contiene molti articoli in buona parte di autori stranieri e si prende atto che la pubblicazione ha oggi raggiunto 12 numeri annui. La

(*) Istituto Lombardo Accademia di Scienze e Lettere, Politecnico Milano, Italia. E-mail: giansacchi.landriani@gmail.com 
scrittura delle formule si diversifica nel tempo. I metodi di calcolo attuali consentono come noto una rapida presentazione esemplificativa. La meccanica analitica acquista il ruolo di indispensabile rigoroso fondamento.

I Rendiconti hanno rappresentato per molti anni la naturale sede scientifica di maestri insigni e di giovani ricercatori. La tematica dei contributi raccolti nella Classe di Scienze Matematiche, Fisiche e Naturali è naturalmente più ampia di quella offerta da Meccanica. A conferma dello stretto rapporto tra AIMETA e Lombardo si può osservare che nel volume del 1966 dei Rendiconti figurano contributi di Maier, Sacchi Landriani, Villaggio, Leo Finzi, Sesini.

Figura indimenticabile, anche perché personaggio di inuguagliabile stile, è Giovanni Bianchi parte attiva di AIMETA, del Lombardo e per molti anni Segretario Generale del CISM di Udine.

Il n $n^{\circ}$ 4/2016 di Meccanica riporta il ricordo, magistralmente tracciato da Del Piero, di Piero Villaggio, molto attivo socio di AIMETA e autore di molti contributi su Meccanica. Si noti che cinque sue memorie dei primi anni '60 figurano sui Rendiconti presentate da Bruno Finzi.

Il legame tra le due istituzioni si è consolidato quando il Lombardo è diventato sede dell'intera collezione di Meccanica.

Prima di dare inizio ai nostri lavori osservo che sono tra noi Giulio Maier che ha disdetto un precedente impegno pur di essere presente, e Jean Salençon, membro straniero dell'Istituto Lombardo già professore di Meccanica all'École Polytechnique di Paris Palaiseau dell'ENPC di Paris e President 2009-2010 de l'Académie des Sciences de l'Institut de France. Siamo grati ad ambedue per la loro presenza.

Il Comitato Scientifico ringrazia: il Presidente Beretta e il Presidente Luchini per la loro disponibilità, la Cancelliere dottoressa Bianchi, il Personale del Lombardo, e tutti voi che siete presenti, certi di una proficua giornata. 